\title{
Differences in mRNA-1273 (Moderna) and BNT162b2 (Pfizer-BioNTech) SARS-CoV-2 vaccine immunogenicity among patients undergoing dialysis
}

\author{
Kevin Yau MD, Christopher T. Chan MD, Kento T. Abe BSc, Yidi Jiang MSc, Mohammad Atiquzzaman PhD, \\ Sarah I. Mullin BSc, Ellen Shadowitz, Lisa Liu, Ema Kostadinovic, Tatjana Sukovic BSc, Anny Gonzalez BSc, \\ Margaret E. McGrath-Chong BScN, Matthew J. Oliver MD MHS, Jeffrey Perl MD SM, Jerome A. Leis MD MSc, \\ Shelly Bolotin MSc PhD MScPH, Vanessa Tran PhD, Adeera Levin MD, Peter G. Blake MB MSc, \\ Karen Colwill PhD, Anne-Claude Gingras PhD, Michelle A. Hladunewich MD MSc
}

Cite as: CMAJ 2022. doi: 10.1503/cmaj.211881; early-released February 3, 2022

\section{Abstract}

Background: Differences in immunogenicity between mRNA SARS-CoV-2 vaccines have not been well characterized in patients undergoing dialysis. We compared the serologic response in patients undergoing maintenance hemodialysis after vaccination against SARS-CoV-2 with BNT162b2 (PfizerBioNTech) and mRNA-1273 (Moderna).

Methods: We conducted a prospective observational cohort study at 2 academic centres in Toronto, Canada, from Feb. 2, 2021 , to July 20,2021 , which included 129 and 95 patients who received the BNT162b2 and mRNA-1273 SARS-CoV-2 vaccines, respectively. We measured SARSCoV-2 immunoglobulin $\mathrm{G}$ antibodies to the spike protein (anti-spike), receptor binding domain (anti-RBD) and nucleocapsid pro- tein (anti-NP) at 6-7 and 12 weeks after the second dose of vaccine and compared those levels with the median convalescent serum antibody levels from 211 controls who were previously infected with SARS-CoV-2.

Results: At 6-7 weeks after 2-dose vaccination, we found that 51 of 70 patients $(73 \%)$ who received BNT162b2 and 83 of 87 (95\%) who received mRNA-1273 attained convalescent levels of anti-spike antibody $(p<0.001)$. In those who received BNT162b2, 35 of 70 (50\%) reached the convalescent level for antiRBD compared with 69 of 87 (79\%) who received mRNA-1273 $(p<0.001)$. At 12 weeks after the second dose, anti-spike and anti-RBD levels were significantly lower in patients who received BNT162b2 than in those who received mRNA-1273. For anti-spike, 70 of 122 patients (57.4\%) who received BNT162b2 maintained the convalescent level versus 68 of 71 (96\%) of those who received mRNA-1273 ( $p<$ 0.001 ). For anti-RBD, 47 of 122 patients (38.5\%) who received BNT162b2 maintained the anti-RBD convalescent level versus 45 of $71(63 \%)$ of those who received mRNA-1273 $(p=0.002)$.

Interpretation: In patients undergoing hemodialysis, mRNA-1273 elicited a stronger humoral response than BNT162b2. Given the rapid decline in immunogenicity at 12 weeks in patients who received BNT162b2, a third dose is recommended in patients undergoing dialysis as a primary series, similar to recommendations for other vulnerable populations.

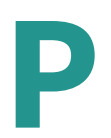

atients with end-stage kidney disease who are receiving maintenance hemodialysis (HD) are at increased risk for severe COVID-19, with mortality rates ranging from $9 \%$ to $28 \% .{ }^{1,2}$ Highly effective vaccines have been developed against SARS-CoV-2, with 94.1\%-95\% efficacy in reducing the risk of severe COVID-19 (D614G strain) as confirmed by 2 large randomized controlled trials; however, these studies included limited numbers of patients with kidney disease., ${ }^{3,4}$ Humoral response to vaccination appears to be heterogeneous in dialysis patients in comparison with the general population, and a review of 35 studies involving dialysis patients found that in the 1-month period after 2-dose vaccination, seroconversion rates ranged from $70 \%$ to $96 \%{ }^{5}$

The BNT162b2 (Pfizer BioNTech) and mRNA-1273 (Moderna) SARS-CoV-2 vaccines are both lipid nanoparticle-encapsulated, nucleoside-modified mRNA encoding for the full-length SARSCoV-2 spike protein stabilized in its prefusion conformation. The BNT162b2 vaccine is administered as a $30 \mu \mathrm{g}$ dose 21 days apart and mRNA- 1273 is administered as a $100 \mu \mathrm{g}$ dose 28 days apart., 
The spike protein and its receptor-binding domain of SARS-CoV-2 are antigens that are targeted by the currently available vaccines and are used as measures of humoral response to vaccination or natural infection. An antibody response to the amount of nucleocapsid protein (NP), which is not targeted by mRNA SARS-CoV-2 vaccines, may be used as a marker of natural exposure to SARS-CoV-2.

Recognition of the high morbidity and mortality from COVID-19 and reduced immunogenicity to vaccination against SARS-CoV-2 in patients undergoing HD has resulted in the prioritization of vaccination of this population in many jurisdictions. ${ }^{1,6}$ However, differences in immunogenicity among SARS-CoV-2 vaccines have not been well characterized in this vulnerable population. Therefore, we conducted a prospective observational study in a cohort of patients undergoing dialysis who received either the mRNA-1273 or BNT162b2 vaccine to evaluate humoral response through comparison of spike and receptor-binding domain antibodies in response to 2 -dose vaccination.

\section{Methods}

\section{Study design and participants}

We conducted a prospective observational cohort study that included patients aged 18 years or older who were undergoing dialysis (including those with previous COVID-19 confirmed by reversetranscription polymerase chain reaction [RT-PCR]) to evaluate SARSCoV-2 antibody response to the 2 available mRNA SARS-CoV-2 vaccines at 2 academic centres (Sunnybrook Health Sciences Centre and University Health Network, Toronto, Ontario). The type of vaccine administered was centre-specific: BNT162b2 was given at Sunnybrook Health Sciences Centre and mRNA-1273 was given at University Health Network. Dialysis patients included those on incentre HD, nocturnal in-centre HD and home HD. We recruited 224 participants between Feb. 2, 2021, and July 20, 2021, at the 2 centres. We obtained written informed consent from all participants.

\section{Serologic assays}

We measured SARS-CoV-2 anti-spike, anti-RBD and anti-NP immunoglobulin $\mathrm{G}$ (IgG) antibodies on an automated enzyme-linked immunosorbent assay (ELISA) platform as reported previously ${ }^{7-9}$ at 6-7 and 12 weeks after 2-dose vaccination. Antibody levels were reported as relative ratios to a synthetic standard included as a calibration curve on each assay plate. We used VHH72-hFc1X7 $(\mathrm{VHH}-$ $72-\mathrm{Fc}$ ) as the synthetic standard for anti-spike and anti-RBD as described previously, ${ }^{10}$ whereas human anti-nucleocapsid IgG (clone HC2003, GenScript, no. A02039) was used for antinucleocapsid. For VHH72-hFc1X7, the llama single-domain monoclonal antibody $\mathrm{VHH}-72$ was expressed as a human Fc fusion: $\mathrm{VHH}-$ 72-hFc1X7 (PDB entry 6WAQ_1). We isolated additional VHHs (NRCoV2-04 and NRCoV2-20) in house from llamas immunized with recombinant SARS-CoV-2 trimeric spike ectodomain SmT1. VHH sequences were fused to an antibody-dependent cell-mediated cytotoxicity attenuated human IgG1 Fc domain (hFc1X7, US patent 2019352 383A1). A table for conversion from relative ratios to the World Health Organization International Standard unit in $B A U / m L$ is provided in Appendix 1, available at www.cmaj.ca/lookup/ doi/10.1503/cmaj.211881/tab-related-content.
We determined thresholds for positivity (seroconversion) by aggregating data from negative controls and calculating the mean plus 3 standard deviations (SDs). Seroconversion thresholds were 0.19, 0.186 and 0.396 for anti-spike, anti-RBD and anti-NP antibodies, respectively. We also compared antibody levels with the median levels of convalescent serum taken 21-115 days after symptom onset in a cohort of 211 patients in the general population (median age $59 \mathrm{yr}$ ) who had mild to severe COVID-19. We considered the median level of antibody in convalescent serum to be a robust antibody response; ${ }^{11}$ medians were $1.38,1.25$ and 1.13 for anti-spike, anti-RBD and anti-NP antibodies, respectively.

\section{Statistical analysis}

We compared baseline characteristics of participants using a $t$ test for continuous variables and $\chi^{2}$ or Fisher exact tests for categorical variables. Anti-spike and anti-RBD levels had skewed distributions and were compared between patients receiving BNT162b2 and mRNA-1273 using the Mann-Whitney test. For patients who had serology results available at both 6-7 and 12 weeks, we compared proportions using the McNemar test. To adjust for imbalance in baseline characteristics, anti-spike and anti-RBD levels at $6-7$ weeks and 12 weeks were transformed by the function $y=$ [relative ratio $-\min$ (relative ratio) +0.001$] /$ [max (relative ratio) $-\max$ (relative ratio) +0.002 ] to be set within the unit interval $[0,1]$. We used the transformed data for $\beta$-regression with the logit link function and adjusted for the following covariates to provide estimates of relative ratios between the 2 study groups: age, sex, immunosuppression, previous solid-organ transplant, coronary artery disease and diabetes mellitus. We selected covariates based upon factors that might plausibly affect immunogenicity or were imbalanced between study groups. $\beta$-Regression is a form of generalized linear model that provides estimates for the dependent variables while modelling changes in variances as mean and precision parameters and allows for modelling of nonnormal distributions. We assessed assumptions for the regression model using analysis of standardized residuals, multicollinearity using the Fisher exact test for binary variables and simple linear regression for continuous variables. We used the logit link function to obtain parameter estimates for the covariates' effects on the transformed antibody levels. We calculated adjusted odds ratios (ORs) using exponentiation of parameter estimates to determine the strength of association between a change in the explanatory variables including vaccine type and anti-spike or anti-RBD levels. All covariates were binary except for age, which was continuous. We performed bootstrapping with 5000 replications to calculate $95 \%$ confidence intervals $(\mathrm{Cls})$ for the median difference in anti-spike and anti-RBD levels at both 6-7 and 12 weeks. We considered a 2-sided $p$ value of 0.05 to be significant. We performed all analyses using $\mathrm{R}$ version 3.6.1 (R Project for Statistical Computing).

\section{Ethics approval}

The study was approved by Mount Sinai Hospital, Sunnybrook Health Sciences Centre and University Health Network (CTO No. 3604). 


\section{Results}

Among 224 patients undergoing dialysis, 129 received the BNT162b2 vaccine and 95 received mRNA-1273. No serious adverse events were reported with either vaccine for any of the study participants. Of those who received BNT162b2, 70 had serology testing performed at 6-7 weeks and 122 had serology testing performed 12 weeks after 2-dose vaccination (Figure 1); these patients had a median age of 72 (interquartile range [IQR] 62-79) years, 41 of 129 (31.7\%) were female and 6 of 129 (4.6\%) had previously had COVID-19 that was confirmed by RT-PCR. Of those who received mRNA-1273, 87 had serology testing performed at 6-7 weeks and 71 had serology testing performed at 12 weeks after 2-dose vaccination; these patients had a median age of 62 (IQR 55-67) years, 25 of 95 (26\%) were female, and 5 of 95 (5.3\%) had a previous SARS-CoV-2 infection. Patients who received BNT162b2 had a higher proportion of diabetes mellitus and coronary artery disease, whereas a higher proportion of patients who received mRNA-1273 had a history of solid organ transplant and were taking immunosuppressive medication (Table 1). We found that serologic evidence of previous SARS-CoV-2 exposure as determined by anti-NP seropositivity was similar between groups: 10 of $87(12 \%)$ who received mRNA-1273 and 12 of $70(17 \%)$ who received $B N T 162 b 2$ were seropositive for anti-NP antibody at $6-7$ weeks $(p=0.3)$, and 4 of $71(6 \%)$ who received mRNA-1273 and 14 of 122 (11.5\%) who received BNT162b2 were seropositive for anti-NP at 12 weeks after vaccination $(p=0.2)$ (Table 2 ).
At 6-7 weeks post 2-dose vaccination, we found that median anti-spike levels were significantly higher for mRNA-1273 (median relative ratio 1.72, IQR 1.68-1.79) than for BNT162b2 (median relative ratio 1.58 , IQR $1.28-1.67 ; p<0.001$ ). We did not find a significant difference in anti-RBD levels (median relative ratio 1.44 , IQR 1.29-1.53, for mRNA-1273 and median relative ratio 1.23, IQR 0.61-1.64, for BNT162b2; $p=0.2$ ). Using the median convalescent serum level as a marker of robust antibody response, among those receiving mRNA-1273, 83 of 87 (95\%) had anti-spike and 69 of $87(79 \%)$ had anti-RBD levels that reached the convalescent serum level at 6-7 weeks after vaccination. This was greater than for BNT162b2 for which 51 of 70 (73\%) had anti-spike and 35 of 70 $(50 \%)$ had anti-RBD levels that reached the convalescent serum level $(p<0.001)$ (Table 2).

At 12 weeks after 2 -dose vaccination, anti-spike levels had declined compared with 6-7 weeks after 2-dose vaccination in those who received BNT162b2 but not in patients who received mRNA-1273 $(p<0.001)$. Anti-spike levels at 12 weeks remained lower in patients who received BNT162b2 (median relative ratio 1.45 , IQR 1.09-1.58) than those who received mRNA-1273 (median relative ratio 1.93 , IQR $1.76-2.02 ; p<0.001$ ). Similarly, anti-RBD levels were lower in patients who received BNT162b2 (median relative ratio 0.89 , IQR $0.36-1.43$ ) than in those who received mRNA-1273 (median relative ratio 1.32, IQR 1.04-1.47; $p=$ 0.002 ) at 12 weeks (Figure 2). Corresponding to the decline in antispike and anti-RBD levels, 70 of 122 patients (57.4\%) who received BNT162b2 had antibody levels above the median convalescent

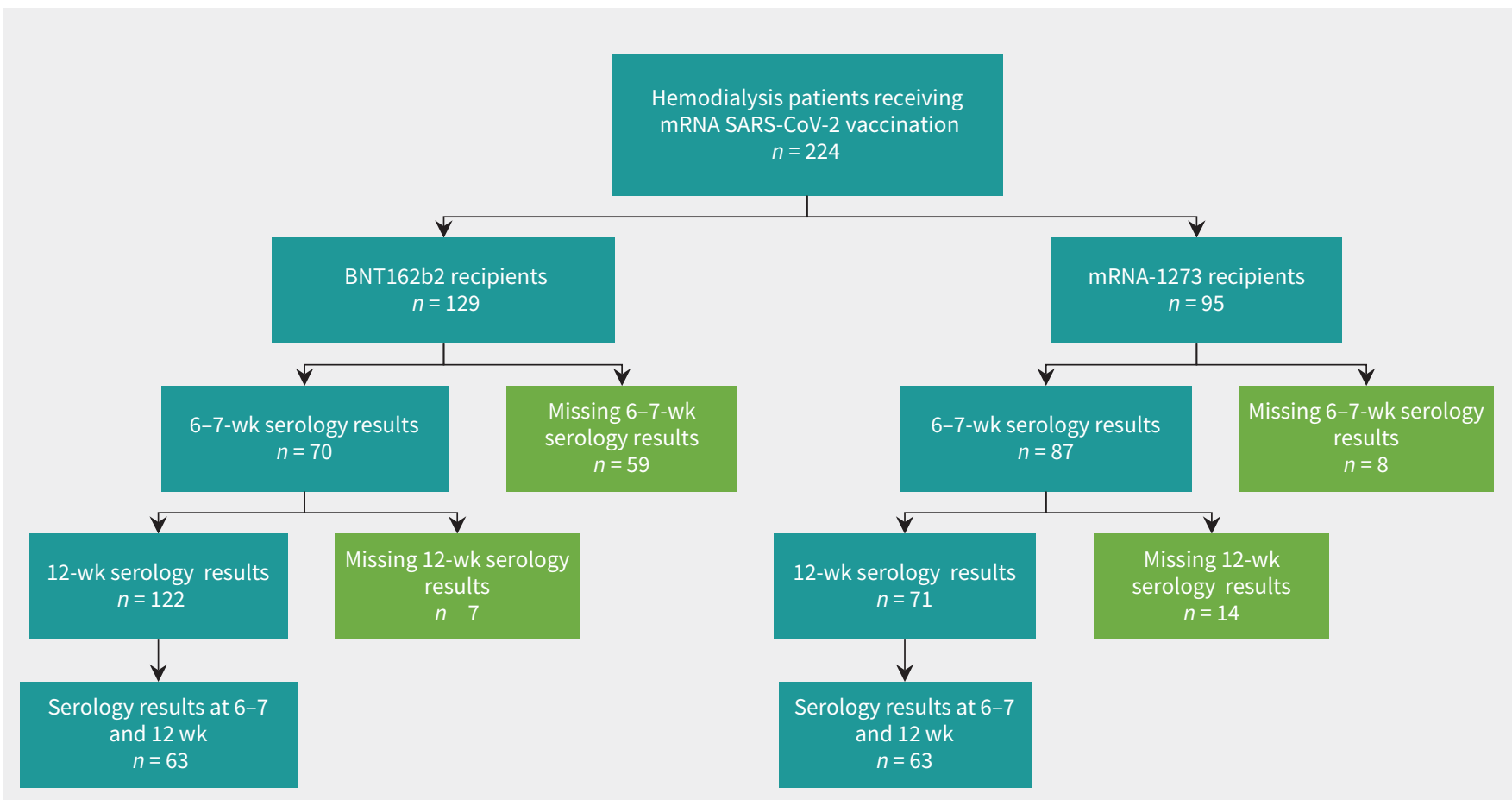

Figure 1: Flow chart of the study cohorts of patients undergoing hemodialysis between Feb. 2, 2021, and July 20, 2021, who were vaccinated against SARS-CoV-2 with 2 doses of either BNT162b2 (Pfizer-BioNTech) or mRNA-1273 (Moderna), and timing of SARS-CoV-2 serologic assessment after 2-dose vaccination. Note: Missing serology results for patients who received BNT162b2 $(n=59)$ at $6-7$ weeks was because of the timing of the start of the study, which led to missing baseline samples at this site. Other missing serology results are reflective of the dialysis population missing scheduled dialysis treatments or because of intercurrent hospital admissions. 
Table 1: Characteristics of patients undergoing hemodialysis who received BNT162 b2 and mRNA-1273 COVID-19 vaccines

\begin{tabular}{|c|c|c|c|}
\hline \multirow[b]{2}{*}{ Characteristic } & \multicolumn{2}{|c|}{ No. $(\%)$ of hemodialysis patients* } & \multirow[b]{2}{*}{$p$ value } \\
\hline & $\begin{array}{c}\text { BNT162b2 vaccine } \\
n=129\end{array}$ & $\begin{array}{c}\text { mRNA-1273 vaccine } \\
\qquad n=95\end{array}$ & \\
\hline Age, yr; median (IQR) & $72(62-79)$ & $62(55-67)$ & $<0.001$ \\
\hline Male sex & $88(68.2)$ & $70(73.7)$ & 0.4 \\
\hline Previous COVID-19† & $6(4.6)$ & $5(5.3)$ & 1.00 \\
\hline Days between doses, median (IQR) & $26(21-35)$ & $30(30-31)$ & $<0.001$ \\
\hline \multicolumn{4}{|l|}{ Comorbidity } \\
\hline Prior solid-organ transplant & $5(4.0)$ & $28(29.5)$ & $<0.001$ \\
\hline Immunosuppressive treatment $\ddagger$ & $10(7.7)$ & $17(17.9)$ & 0.02 \\
\hline Diabetes mellitus & $72(55.8)$ & $33(34.7)$ & 0.002 \\
\hline Coronary artery disease & $52(40.3)$ & $14(14.7)$ & $<0.001$ \\
\hline Congestive heart failure & $35(27.1)$ & $21(22.1)$ & 0.4 \\
\hline Cerebrovascular disease & $17(13.2)$ & $9(9.5)$ & 0.4 \\
\hline Hypertension & $121(93.7)$ & $84(88.4)$ & 0.15 \\
\hline
\end{tabular}

Table 2: Relative ratios, seroconversion and proportion of patients attaining the median convalescent serum levels for SARS-CoV-2 antibodies after BNT162b2 or mRNA-1273 vaccination

\begin{tabular}{|c|c|c|c|c|c|c|c|}
\hline \multirow{2}{*}{$\begin{array}{l}\text { Weeks after } \\
\text { second dose }\end{array}$} & \multirow[b]{2}{*}{ Antibody } & \multicolumn{2}{|c|}{ Relative ratio, median (IQR)* } & \multicolumn{2}{|c|}{$\begin{array}{l}\text { No. (\%) of patients with } \\
\text { seroconversiont }\end{array}$} & \multicolumn{2}{|c|}{$\begin{array}{c}\text { No. }(\%) \text { of patients who reached } \\
\text { convalescent level }\end{array}$} \\
\hline & & BNT162b2 & mRNA-1273 & BNT162b2 & mRNA-1273 & BNT162b2 & mRNA-1273 \\
\hline \multirow[t]{3}{*}{$6-7$} & Anti-spike & $1.58(1.28-1.67)$ & $1.72(1.68-1.79)$ & $\begin{array}{l}68(97) \\
n=70\end{array}$ & $\begin{array}{l}84(97) \\
n=87\end{array}$ & $\begin{array}{l}51(73) \\
n=70\end{array}$ & $\begin{array}{l}83(95) \\
n=87\end{array}$ \\
\hline & Anti-RBD & $1.23(0.61-1.64)$ & $1.44(1.29-1.53)$ & $\begin{array}{l}62(89) \\
n=70\end{array}$ & $\begin{array}{l}84(97) \\
n=87\end{array}$ & $\begin{array}{l}35(50) \\
n=70\end{array}$ & $\begin{array}{l}69(79) \\
n=87\end{array}$ \\
\hline & Anti-NP & - & - & $\begin{array}{l}12(17) \\
n=70\end{array}$ & $\begin{array}{l}10(12) \\
n=87\end{array}$ & $\begin{array}{c}6(9) \\
n=70\end{array}$ & $\begin{array}{c}4(5) \\
n=87\end{array}$ \\
\hline \multirow[t]{3}{*}{12} & Anti-spike & 1.45 (1.09-1.58) & $1.93(1.76-2.02)$ & $\begin{array}{c}117(95.9) \\
n=122\end{array}$ & $\begin{array}{c}68(96) \\
n=71\end{array}$ & $\begin{array}{l}70(57.4) \\
n=122\end{array}$ & $\begin{array}{c}68(96) \\
n=71\end{array}$ \\
\hline & Anti-RBD & $0.89(0.36-1.43)$ & $1.32(1.04-1.47)$ & $\begin{array}{c}107(87.7) \\
n=122\end{array}$ & $\begin{array}{l}67(94) \\
n=71\end{array}$ & $\begin{array}{l}47(38.5) \\
n=122\end{array}$ & $\begin{array}{c}45(63) \\
n=71\end{array}$ \\
\hline & Anti-NP & - & - & $\begin{array}{l}14(11.5) \\
n=122\end{array}$ & $\begin{array}{c}4(6) \\
n=71\end{array}$ & $\begin{array}{c}7(5.7) \\
n=122\end{array}$ & $\begin{array}{c}2(3) \\
n=71\end{array}$ \\
\hline $\begin{array}{l}\text { Note: IQR = interc } \\
\text { *We did not repo } \\
\text { †Antibody levels } \\
\text { anti-RBD and ant } \\
\text { †The median leve } \\
\text { antibody respons }\end{array}$ & $\begin{array}{l}\mathrm{NP}=\text { nucleoca } \\
\text { tistics for anti } \\
\text { s relative ratio } \\
\text { s, respectively } \\
\text { convalescent } \\
\text { nd } 1.13 \text { for ant }\end{array}$ & $\begin{array}{l}\text { protein, RBD = recep } \\
\text { ecause it was used a } \\
\text { ynthetic standards. } S \\
\text { was from a cohort } \\
\text {, anti-RBD and anti }\end{array}$ & $\begin{array}{l}\text { or binding domain. } \\
\text { s binary measure of } n \\
\text { eroconversion thresho } \\
\text { f } 211 \text { patients in the ge } \\
\text { NP antibodies, respect }\end{array}$ & $\begin{array}{l}\text { al SARS-CoV-2 i } \\
\text { vels representec }\end{array}$ & $\begin{array}{l}\text { on. } \\
\text { sitive test and we } \\
\text { vious COVID-19o }\end{array}$ & $0.19,0.186$ and 0.39 & $\begin{array}{l}\text { anti-spike, } \\
\text { ted a robust }\end{array}$ \\
\hline
\end{tabular}

level of anti-spike compared with 68 of 71 (96\%) who received mRNA-1273 $(p<0.001)$ at 12 weeks. At 12 weeks, 47 of 122 patients (38.5\%) who received BNT162b2 passed the median convalescent serum level of anti-RBD compared with 45 of 71 patients $(63 \%)$ who received mRNA-1273 $(p=0.002)$. Three patients who received $B N T 162$ b2 had SARS-CoV-2 infection confirmed by RTPCR after 2-dose vaccination at 4, 11 and 71 days after the second dose, and 2 of these patients required admission to hospital. No patients who received mRNA-1273 contracted COVID-19 during the study period.

We conducted a longitudinal analysis of patients with serology results $(n=126)$ at both $6-7$ and 12 weeks after 2-dose vaccination that confirmed greater immunogenicity with mRNA-1273 than with BNT162b2 (Appendix 2, available at www.cmaj.ca/lookup/ 


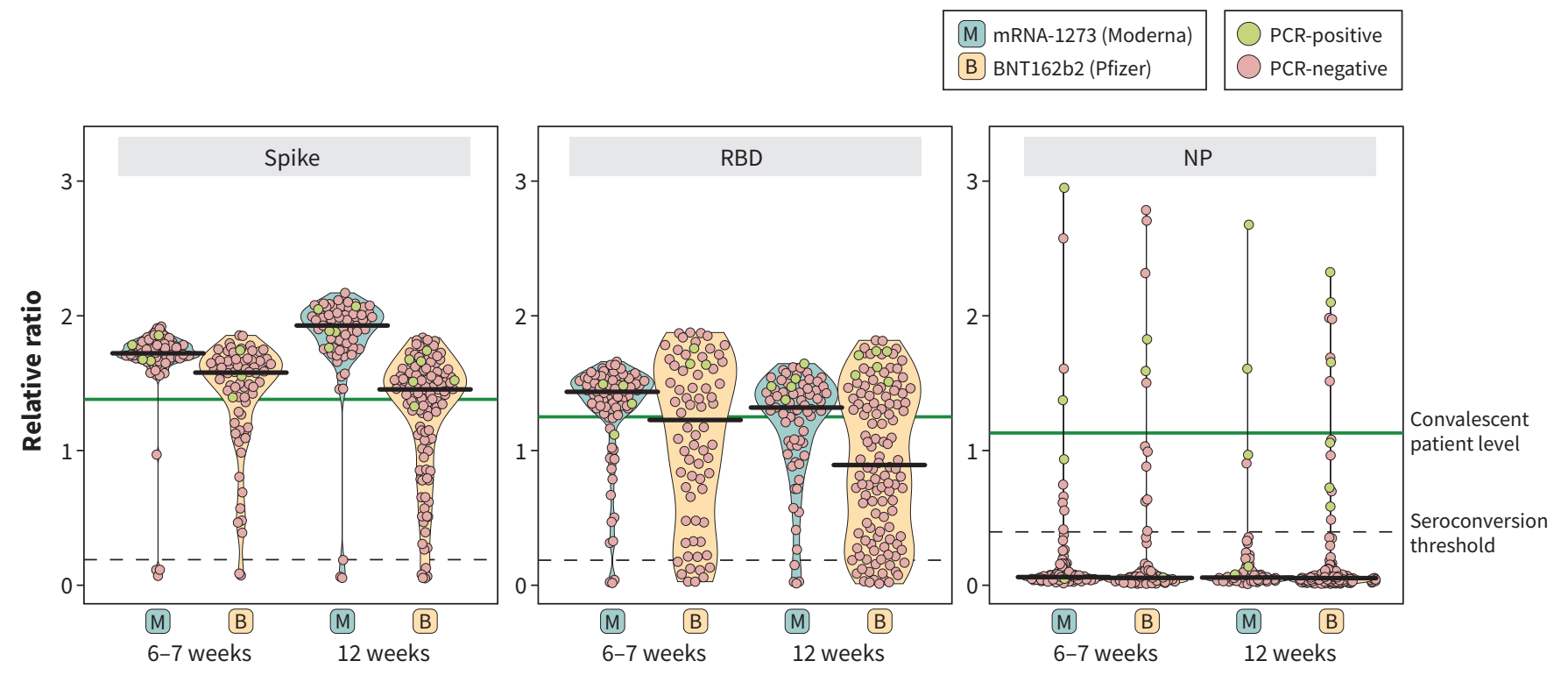

Figure 2: SARS-CoV-2 immunoglobulin G spike, RBD and NP antibody responses in patients undergoing hemodialysis after mRNA-1273 (Moderna; $n=95$ ) or BNT162b2 (Pfizer-BioNTech; $n=129$ ) vaccination. Dots represent individual serum samples collected at $6-7$ and 12 weeks after 2-dose vaccination. Patients with previous SARS-CoV-2 infection confirmed by RT-PCR are indicated in green. Antibody levels are reported as relative ratios to synthetic standards. Seroconversion threshold levels represent a positive test and are $0.19,0.186$ and 0.396 for anti-spike, anti-RBD and anti-NP antibodies, respectively, and are indicated by the broken line for each antibody. Median antibody levels for each antigen are indicated by the solid black lines. The median level of antigen in convalescent serum is from a cohort of 211 patients in the general population with previous COVID-19 of all severities, represents a robust antibody response (1.38, 1.25 and 1.13 for anti-spike, anti-RBD, and anti-NP antibodies, respectively) and is indicated by the green line for each antibody. Note: NP = nucleocapsid protein, RBD = receptor binding domain, $\mathrm{RT}-\mathrm{PCR}=$ reverse transcription-polymerase chain reaction.

doi/10.1503/cmaj.211881/tab-related-content), although imbalance in a number of baseline characteristics persisted (Appendix 3, available at www.cmaj.ca/lookup/doi/10.1503/cmaj.211881/tab -related-content). Our results were similar when we excluded patients with previous COVID-19 that was confirmed by RT-PCR or anti-NP seroconversion during the study period from the analysis (Appendix 4 and Appendix 5, available at www.cmaj.ca/lookup/ doi/10.1503/cmaj.211881/tab-related-content), when we analyzed patients who were taking immunosuppressive medications or who had a history of solid-organ transplant separately (Appendix 6, available at www.cmaj.ca/lookup/doi/10.1503/cmaj.211881/tab -related-content) or when we compared by age (Figure 3). Standardized Pearson residuals in the $\beta$-regression models were appropriately zero centred, which supports a linear relation between the covariates and the antibody levels (Appendix 7, available at www. cmaj.ca/lookup/doi/10.1503/cmaj.211881/tab-related-content). At 6-7 weeks after vaccination, we found that mRNA-1273 was associated with higher anti-spike levels (unadjusted OR 1.79, 95\% Cl 1.30-2.47; adjusted OR 2.04, 95\% Cl 1.39-3.01), whereas anti-RBD levels were not significantly higher (unadjusted OR $1.15,95 \% \mathrm{Cl}$ 0.81-1.63; adjusted OR $1.44,95 \% \mathrm{Cl} 0.96-2.16)$ than BNT162b2 (Table 3). This became more pronounced at 12 weeks: mRNA-1273 was associated with higher anti-spike levels (unadjusted OR 2.94, 95\% Cl 2.17-3.97; adjusted OR 3.34, 95\% Cl 2.42-4.62) and higher anti-RBD levels (unadjusted OR 1.46, 95\% Cl 1.05-2.03; adjusted OR 1.55, 95\% Cl 1.09-2.21). We confirmed the higher anti-spike and anti-RBD levels with mRNA-1273 using bootstrapping with 5000 replications (Appendix 8 and Appendix 9, available at www. cmaj.ca/lookup/doi/10.1503/cmaj.211881/tab-related-content).

\section{Interpretation}

We compared the SARS-CoV-2 humoral response after vaccination in 224 patients receiving maintenance $H D$ and found that mRNA-1273 elicited greater immunogenicity than BNT162b2 at 6-7 weeks after vaccination, and antibody levels were better maintained with mRNA-1273 at 12 weeks. Although we measured only the serologic response to vaccination, a humoral response is known to be necessary to obtain an adequate cellular response. ${ }^{12,13}$ The decline in SARS-CoV-2 antibodies at 12 weeks after vaccination in those who received BNT162b2 is concerning because levels of anti-spike and anti-RBD generally correlate with levels of neutralizing antibody, which have been inferred to provide protection against symptomatic SARS-CoV-2 infection. ${ }^{11}$ Our results are consistent with those of a study involving 2367 patients undergoing dialysis in the United States at 28-60 days after vaccination: $22.7 \%$ of those who received BNT162 b2 had no detectable anti-RBD IgG level compared with $9.4 \%$ of those who received mRNA-1273 (levels measured using a semiquantitative commercial assay). ${ }^{14}$

Evaluations of the differences in immunogenicity between the mRNA vaccines are limited. A 2021 study involving 1647 health care workers in Belgium reported that SARS-CoV-2 anti-spike levels were significantly higher with mRNA-1273 than with BNT162b2 at $6-10$ weeks after 2 -dose vaccination. ${ }^{15}$ The authors speculated that this difference could be explained by the higher mRNA content in mRNA-1273 and the slightly longer boost period (28 v. $21 \mathrm{~d}) .{ }^{15} \mathrm{~A} 2021$ study involving residents and health care workers at long-term care homes in Ontario reported stronger humoral 

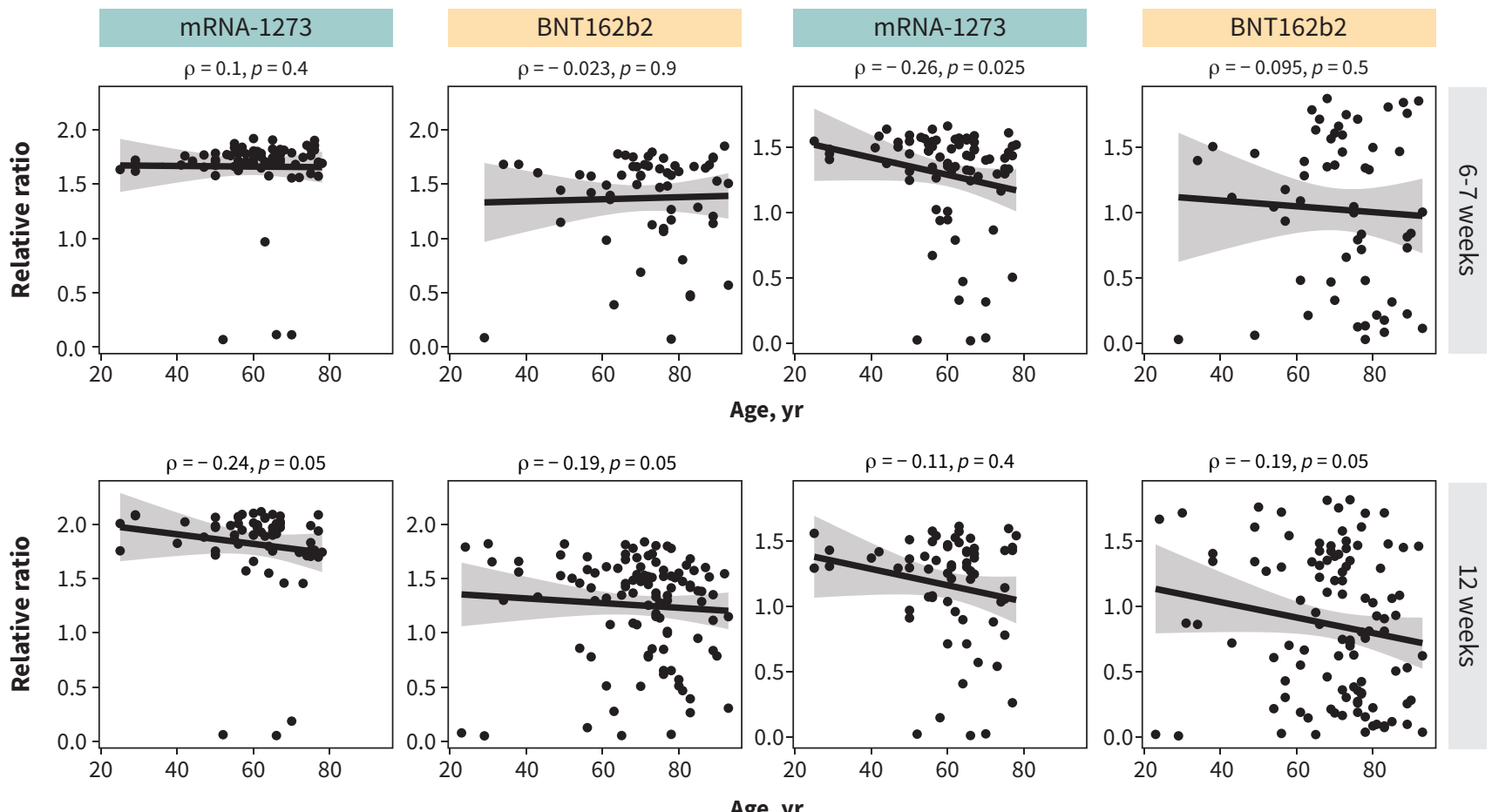

Age, yr

B

RBD, 0.00156 dilution
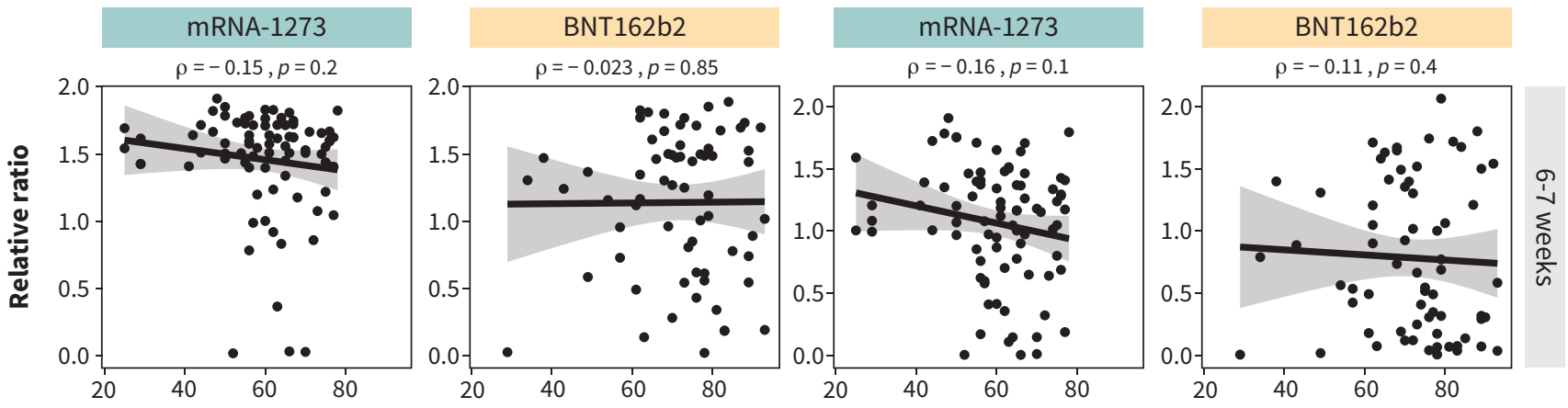

Age, yr
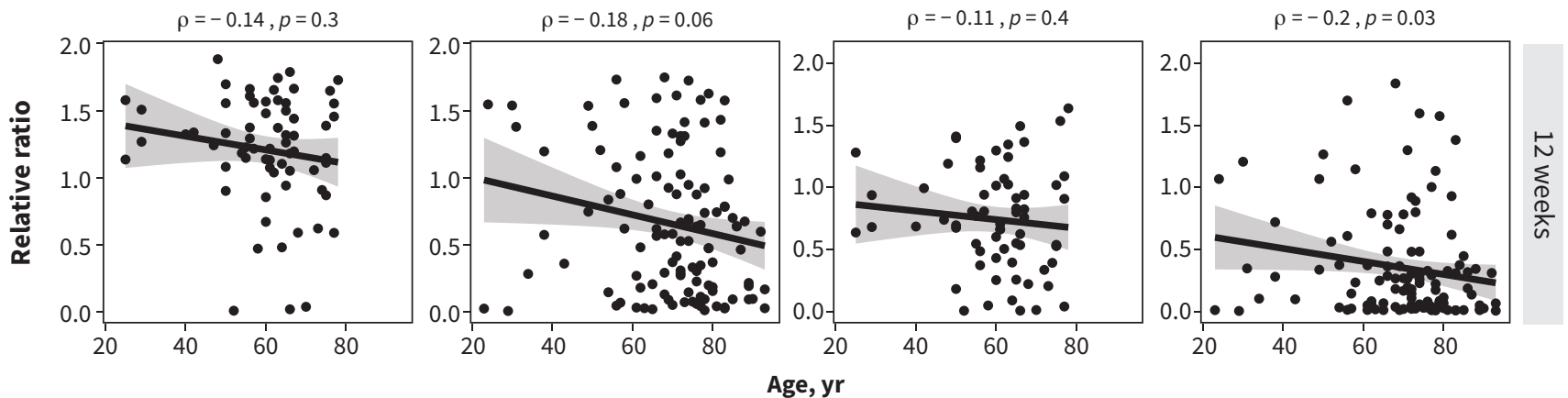

Figure 3: SARS-CoV-2 immunoglobulin G spike and RBD antibody response in patients undergoing hemodialysis after vaccination with mRNA-1273 (Moderna; $n=95$ ) or BNT162b2 (Pfizer-BioNTech; $n=129$ ), by age. Results are shown at the (A) 0.00625 dilution and (B) 0.00156 dilution levels. Linear regression lines with 95\% confidence intervals (grey) are shown with corresponding Spearman $\rho$ and $p$ values. Dots represent individual serum samples collected at 6-7 and 12 weeks after 2-dose vaccination. Antibody levels are reported as relative ratios to synthetic standards. Seroconversion threshold levels represent positive tests and are 0.19 and 0.186 for anti-spike and anti-RBD antibodies, respectively. Note: RBD $=$ receptor binding domain. 
Table 3: Multivariable $\beta$-regression models of predictors of anti-spike or anti-RBD levels at 6-7 and 12 weeks after 2-dose vaccination

\begin{tabular}{|c|c|c|c|c|c|c|}
\hline Analysis & Variable & $\begin{array}{l}\text { Unadjusted OR } \\
\qquad(95 \% \mathrm{Cl})\end{array}$ & $\begin{array}{l}\text { Adjusted OR } \\
(95 \% \mathrm{CI})\end{array}$ & $\begin{array}{l}\text { Parameter } \\
\text { estimate }\end{array}$ & Standard error & $p$ value \\
\hline & & \multicolumn{5}{|c|}{ 6-7 weeks after vaccination } \\
\hline \multirow[t]{7}{*}{ Anti-spike } & mRNA-1273 (ref. BNT162b2) & $1.79(1.30-2.47)$ & $2.04(1.39-3.01)$ & 0.72 & 0.2 & $<0.001$ \\
\hline & Age, yr & - & $1.00(0.99-1.01)$ & 0 & 0.01 & 0.704 \\
\hline & Sex (ref. female) & - & $0.69(0.49-0.98)$ & -0.37 & 0.17 & 0.036 \\
\hline & Immunosuppressive treatment & - & $0.36(0.22-0.60)$ & -1.03 & 0.26 & $<0.001$ \\
\hline & Previous solid-organ transplant & - & $1.30(0.79-2.16)$ & 0.27 & 0.26 & 0.305 \\
\hline & Diabetes & - & $1.19(0.85-1.68)$ & 0.18 & 0.17 & 0.31 \\
\hline & Coronary artery disease & - & $0.84(0.57-1.24)$ & -0.17 & 0.2 & 0.382 \\
\hline \multirow[t]{8}{*}{ Anti-RBD } & mRNA-1273 (ref. BNT162b2) & $1.15(0.81-1.63)$ & $1.44(0.96-2.16)$ & 0.36 & 0.21 & 0.081 \\
\hline & Age, yr & - & $0.99(0.98-1.00)$ & -0.01 & 0.01 & 0.13 \\
\hline & Sex (ref. female) & - & $0.55(0.38-0.79)$ & -0.6 & 0.19 & 0.001 \\
\hline & Immunosuppressive treatment & - & $0.26(0.15-0.45)$ & -1.36 & 0.28 & $<0.001$ \\
\hline & Previous solid-organ transplant & - & $1.48(0.86-2.52)$ & 0.39 & 0.27 & 0.154 \\
\hline & Diabetes & - & $1.40(0.97-2.01)$ & 0.33 & 0.19 & 0.072 \\
\hline & Coronary artery disease & - & $1.20(0.80-1.81)$ & 0.18 & 0.21 & 0.38 \\
\hline & & \multicolumn{5}{|c|}{12 weeks after vaccination } \\
\hline \multirow[t]{7}{*}{ Anti-spike } & mRNA-1273 (ref. BNT162b2) & $2.94(2.17-3.97)$ & $3.34(2.42-4.62)$ & 1.21 & 0.16 & $<0.001$ \\
\hline & Age, yr & - & $0.99(0.98-1.00)$ & -0.01 & 0.01 & 0.075 \\
\hline & Sex (ref. female) & - & $0.77(0.57-1.03)$ & -0.26 & 0.15 & 0.082 \\
\hline & Immunosuppressive treatment & - & $0.28(0.17-0.47)$ & -1.26 & 0.25 & $<0.001$ \\
\hline & Previous solid-organ transplant & - & $0.98(0.59-1.64)$ & -0.02 & 0.26 & 0.948 \\
\hline & Diabetes & - & $1.14(0.86-1.52)$ & 0.14 & 0.14 & 0.348 \\
\hline & Coronary artery disease & - & $1.10(0.80-1.52)$ & 0.1 & 0.16 & 0.558 \\
\hline \multirow[t]{7}{*}{ Anti-RBD } & mRNA-1273 (ref. BNT162b2) & $1.46(1.05-2.03)$ & $1.55(1.09-2.21)$ & 0.44 & 0.18 & 0.014 \\
\hline & Age, yr & - & $0.98(0.97-1.00)$ & -0.02 & 0.01 & 0.006 \\
\hline & Sex (ref. female) & - & $0.69(0.49-0.97)$ & -0.37 & 0.17 & 0.035 \\
\hline & Immunosuppressive treatment & - & $0.29(0.17-0.51)$ & -1.23 & 0.29 & $<0.001$ \\
\hline & Previous solid-organ transplant & - & $1.10(0.62-1.95)$ & 0.1 & 0.29 & 0.743 \\
\hline & Diabetes & - & $0.98(0.71-1.35)$ & -0.02 & 0.16 & 0.899 \\
\hline & Coronary artery disease & - & $1.35(0.93-1.95)$ & 0.3 & 0.19 & 0.111 \\
\hline
\end{tabular}

Note: $\mathrm{Cl}=$ confidence interval, $\mathrm{OR}=$ odds ratio, $\mathrm{RBD}=$ receptor binding domain, ref. = reference category. We transformed anti-spike and anti-RBD levels to be expressed between an interval of 0 and 1 with the following function: $y=$ [relative ratio - $\min ($ relative ratio) +0.001 ] / [max (relative ratio) - max (relative ratio) +0.002 ]. All available serology results were used: BNT162b2 at 6-7 weeks $(n=70)$, mRNA-1273 at $6-7$ weeks $(n=87)$, BNT162b2 at 12 weeks $(n=122)$ and mRNA-1273 at 12 weeks $(n=71)$.

response with mRNA-1273 than with BNT162b2 vaccine. ${ }^{16}$ Residents of long-term care who were administered BNT162b2 produced 3.89-fold fewer neutralizing antibodies against wild-type D614G SARS-CoV-2 using a spike-pseudotyped lentivirus assay than those who received mRNA-1273, which was further reduced by 4.25-fold against the B.1.617.2 (Delta) variant. ${ }^{16}$

Waning antibody levels have been observed in the general population, in particular for those receiving 2 doses of BNT162b2 at the suggested 21-day interval. For example, a 2021 study of antibody titre decay after vaccination against SARS-CoV-2 in Israel reported that $16.1 \%$ of people vaccinated with 2 doses of BNT162b2 had anti-spike levels below the seropositivity threshold at 6 months. ${ }^{17}$ Booster dosages in older adults have been shown to reduce the risk of breakthrough SARS-CoV-2 infections in this population ${ }^{18}$ and, therefore, have now been recommended in Canada for those older than 18 years of age. In the transplant population, a third dose of vaccine was also successful in improving immunogenicity, which led to broad approval of a 3-dose series for recipients of solid-organ transplants in Canada. ${ }^{19}$

Patients undergoing dialysis have impaired humoral response compared with healthy controls and frequently require booster dosages in the setting of vaccination against hepatitis B. Given reduced immunogenicity after vaccination and a high risk for severe COVID-19, patients undergoing dialysis in France, Israel and the US were routinely administered a third dose of SARS-CoV-2 vaccine, which enhanced humoral response, especially in those who 
responded poorly to 2 doses of vaccine..$^{20,21}$ In a 2021 prospective observational study conducted in France involving a cohort of patients receiving maintenance $\mathrm{HD}$, a third dose of BNT162b2 was shown to achieve seroconversion in more than half of the patients who were previously seronegative after 2 doses..$^{22}$ In Canada, a third dose of SARS-CoV-2 mRNA vaccine has been approved for dialysis patients. In Ontario, $74 \%$ of vaccinated patients undergoing dialysis received the BNT162b2 vaccine, which is of concern given the rapid decrement in immunogenicity at 12 weeks among patients receiving the BNT162b2 vaccine in our study. Furthermore, HD units are at risk for COVID-19 outbreaks because of the challenges in practising physical distancing in health care settings, and breakthrough infections have been described in HD patients with low antibody titres..$^{23,24}$ Although we observed 3 cases of COVID-19 after 2-dose vaccination only in the BNT162b2 group in our study, 2 of these cases occurred less than 14 days after the second dose, and breakthrough infections require further evaluation in larger studies. However, a 2021 US study of 56 breakthrough infections in patients undergoing dialysis found that those with anti-RBD IgG levels lower than $218 \mathrm{BAU} / \mathrm{mL}$ were at 11.6 times higher risk for breakthrough infection. ${ }^{25}$

Real-world data support potential differences in effectiveness between SARS-CoV-2 mRNA vaccines. A large observational study at the Mayo Clinic Health System that involved a cohort of matched vaccinated patients found that, although the effectiveness of mRNA-1273 and BNT162b2 was similar in early 2021, after the emergence of the B.1.617.2 (Delta) variant in July 2021, vaccination with mRNA-1273 conferred a 2-fold risk reduction against breakthrough infection compared with BNT162b2. ${ }^{26}$ Further population-based studies will be required to corroborate whether the differences in immunogenicity correspond to decreased vaccine effectiveness, but pending these data, immunogenicity has already been used to guide vaccine policy decisions in other atrisk groups, including older adults and transplant recipients.

\section{Limitations}

Our study was limited by its observational design, which resulted in differences in several baseline characteristics between the 2 cohorts, and a sample size that was based on convenience sampling during the study period. However, after adjustment for potential confounders with regression analysis, the finding of greater immunogenicity with mRNA-1273 compared with BNT162b2 persisted. Patients who received BNT162b2 in our study were older, which reflects earlier onset of the study when older patients were being prioritized for vaccination. This was counterbalanced by usage of immunosuppressive medication among the mRNA-1273 recipients, which was associated with lower antibody levels. Although antibody levels are a useful marker of immunogenicity, ${ }^{11}$ and it has been shown that antibody levels correlate with a reduction in severe SARS-CoV-2 infections, ${ }^{27}$ the antibody level required for protection from COVID-19 is currently unknown. ${ }^{28,29}$ In addition, the definition of robust immunity in our study was a surrogate marker that was defined as reaching the median antibody level in convalescent serum. Although convalescent serum may be a useful comparator, ongoing studies will be required to confirm the utility of convalescent levels and their correlation with vaccine effectiveness.

\section{Conclusion}

We found greater immunogenicity with the mRNA-1273 vaccine than the BNT162b2 vaccine in patients undergoing dialysis, with rapid decline in immunogenicity at 12 weeks after 2-dose vaccination in patients who received BNT162b2. In light of these findings, a timely third dose of SARS-CoV-2 vaccine in this vulnerable population is recommended.

\section{References}

1. Taji L, Thomas D, Oliver MJ, et al. COVID-19 in patients undergoing long-term dialysis in Ontario. CMAJ 2021;193:E278-E284.

2. Chan L, Jaladanki SK, Somani S, et al. Outcomes of patients on maintenance dialysis hospitalized with COVID-19. Clin J Am Soc Nephrol 2021;16:452-5.

3. Baden LR, El Sahly HM, Essink B, et al. Efficacy and safety of the mRNA-1273 SARS-CoV-2 vaccine. N Engl J Med 2021 Feb 4;384:403-16.

4. Polack FP, Thomas SJ, Kitchin N, et al. Safety and efficacy of the BNT162b2 mRNA COVID-19 vaccine. N Engl J Med 2020;383:2603-15.

5. Carr EJ, Kronbichler A, Graham-Brown M, et al. Review of early immune response to SARS-CoV-2 vaccination among patients with CKD. Kidney Int Rep 2021;6:2292-304.

6. Yau K, Abe KT, Naimark D, et al. Evaluation of the SARS-CoV-2 antibody response to the BNT162b2 vaccine in patients undergoing dialysis. JAMA Netw Open 2021;4:e2123622.

7. Abe KT, Li Z, Samson R, et al. A simple protein-based surrogate neutralization assay for SARS-CoV-2. JCI Insight 2020;5:e142362.

8. Isho B, Abe KT, Zuo M, et al. Persistence of serum and saliva antibody responses to SARS-CoV-2 spike antigens in COVID-19 patients. Sci Immunol 2020;5:eabe5511.

9. Colwill K, Galipeau Y, Stuible M, et al. A "Made-in-Canada"; serology solution for profiling humoral immune responses to SARS-CoV-2 infection and vaccination. medRxiv 2021 Oct. 26 [preprint]. doi: https://doi.org/10.1101/2021.10.25.21265476.

10. Wrapp D, De Vlieger D, Corbett KS, et al. Structural basis for potent neutralization of betacoronaviruses by single-domain camelid antibodies. Cell 2020; 181:1004-1015.e15.

11. Khoury DS, Cromer D, Reynaldi A, et al. Neutralizing antibody levels are highly predictive of immune protection from symptomatic SARS-CoV-2 infection. Nat Med 2021;27:1205-11.

12. Bartsch YC, Fischinger S, Siddiqui SM, et al. Discrete SARS-CoV-2 antibody titers track with functional humoral stability. Nat Commun 2021;12:1018.

13. McMahan K, Yu J, Mercado NB, et al. Correlates of protection against SARSCoV-2 in rhesus macaques. Nature 2021;590:630-4.

14. Garcia P, Anand S, Han J, et al. COVID-19 vaccine type and humoral immune response in patients receiving dialysis. J Am Soc Nephrol 2022;33:33-7.

15. Steensels D, Pierlet N, Penders J, et al. Comparison of SARS-CoV-2 antibody response following vaccination with BNT162b2 and mRNA-1273. JAMA 2021; 326:1533-5.

16. Abe KT, Hu Q, Mozafarihashijin M, et al. Neutralizing antibody responses to SARSCoV-2 variants in vaccinated Ontario long-term care home residents and workers. medRxiv 2021 Aug. 6 [preprint]. doi: https://doi.org/10.1101/2021.08.06.21261721.

17. Israel A, Shenhar Y, Green I, et al. Large-scale study of antibody titer decay following BNT162b2 mRNA vaccine or SARS-CoV-2 infection. medRxiv 2021 Aug. 21 [preprint]. doi: 10.1101/2021.08.19.21262111..

18. Bar-On YM, Goldberg Y, Mandel M, et al. Protection of BNT162b2 vaccine booster against Covid-19 in Israel. N Engl J Med 2021;385:1393-400.

19. Kamar N, Abravanel F, Marion O, et al. Three doses of an mRNA COVID-19 vaccine in solid-organ transplant recipients. N Engl J Med 2021;385:661-2.

20. Ducloux D, Colladant M, Chabannes M, et al. Humoral response after 3 doses of the BNT162b2 mRNA COVID-19 vaccine in patients on hemodialysis. Kidney Int 2021;100:702-4.

21. Frantzen L, Thibeaut S, Moussi-Frances J, et al. COVID-19 vaccination in haemodialysis patients: good things come in threes ... . Nephrol Dial Transplant 2021; 36:1947-9.

22. Dekervel M, Henry N, Torreggiani M, et al. Humoral response to a third injection of BNT162b2 vaccine in patients on maintenance haemodialysis. Clin Kidney $\mathrm{J}$ 2021;14:2349-55.

23. Manley HJ, Aweh GN, Hsu CM, et al. SARS-CoV-2 vaccine effectiveness and breakthrough infections in maintenance dialysis patients. medRxiv 2021 Sept. 19 [preprint]. doi: https://doi.org/10.1101/2021.09.24.21264081.

24. Yau K, Muller MP, Lin M, et al. COVID-19 outbreak in an urban hemodialysis unit. Am J Kidney Dis 2020;76:690-695.e1. 
25. Anand S, Montez-Rath ME, Han J, et al. SARS-CoV-2 vaccine antibody response and breakthrough infection in patients receiving dialysis. Ann Intern Med 2021;M21-4176.

26. Puranik A, Lenehan PJ, Silvert E, et al. Comparison of two highly effective mRNA vaccines for COVID-19 during periods of Alpha and Delta variant prevalence. medRxiv 2021 Aug. 8 [preprint]. doi: https://doi.org/10.1101/2021.08.06. 21261707 .

Competing interests: Mohammad Atiquzzaman and Adeera Levein are employees of the BC Renal Agency. Matthew Oliver and Michelle Hladunewich are contracted medical leads at Ontario Renal Network, Ontario Health. Matthew Oliver is the owner of Oliver Medical Management Inc., which licenses Dialysis Management Analysis and Reporting (DMAR) System software. He holds a Canadian patent for DMAR System, has received speaker fees from Baxter Healthcare and participated on advisory boards for Janssen and Amgen. Jeffrey Perl has received consultant fees from Baxter Healthcare, US Renal Care, Davita Healthcare Partners, Otsuka Canada and AstraZeneca Canada, outside the submitted work; grants from the Agency for Healthcare Research and Quality grant support; and speaker fees from Baxter Healthcare, Fresenius Medical Care USA, AstraZeneca, Davita Healthcare Partners and US Renal Care. Shelly Bolotin has received funding from the Canadian Institutes of Health Research (CIHR), the Canadian Immunization Research Network, the COVID-19 Immunity Task Force and the Public Health Agency of Canada, outside the submitted work. She is a member of the Canadian Immunization Research Network Management Committee and the COVID-19 Immunity Task Force Leadership Group. Vanessa Tran reports that Public Health Ontario received funding from the Public Health Agency of Canada and test kits from the Canadian Immunity Task Force for COVID-19 serosurveillance studies. Public Health Ontario is also involved in a COVID-19 mix-and-match vaccine clinical trial. Shelly Bolotin and Vanessa Tran are employees of Public Health Ontario. Adeera Levin is the Lead Integrated Clinical and Academic Networks for Providence Health Care. He has participated on the boards of the National Institute of Diabetes and Digestive and Kidney Diseases, National Institutes of Health and_Australian Kidney Clinical Trials Network. He has a leadership or fiduciary role for the International Society of Nephrology (ISN) Advocacy Group, ISN International CKDu Consortium, ISN Research Committee, and the BC Renal Agency. He has received honoraria from GSK, Takeda, Reata, Astra Zeneca and Janssen; and payment for expert testimony from Osler. Peter Blake is the medical director at the Ontario Renal Network, Ontario Health, and has received speaker fees from Baxter Global. Anne-Claude Gingras is a member of the working parties (testing and immunology) of the COVID-19 Immunity Task Force, is the chair of the CIHR Institute of Genetics Institute Advisory Board is a member of the National Research Council of Canada Human Health Therapeutics Board and is the Pillar lead, Functional Genomics and Structure-Function at CoVaRR-Net. Michelle Hladunewich has received grants from Pfizer, lonis, Chemocentryx, Calliditas and Roche; consultant fees from Alynylam; and royalties from UpToDate, outside the submitted work. Jerome Leis has received payments for expert testimony from the Ontario Hospital Association and the Ministry of the Attorney General of Ontario and has a leadership role with Choosing Wisely Canada. Kevin Yau has received speaker fees from AstraZeneca. No other competing interests were declared.

This article has been peer reviewed.

Affiliations: Division of Nephrology (Yau, Mullin, Shadowitz, Liu, Kostadinovic, Sukovic, Gonzalez, Oliver, Hladunewich) and Division of Infectious Diseases (Leis), Department of Medicine, Sunnybrook Health Sciences Centre, Temerty Faculty of Medicine, University of Toronto; Division of Nephrology (Yau, Chan, McGrath-Chong), Department of Medicine, Temerty Faculty of Medicine, University of Toronto; Department of Molecular Genetics (Abe, Gingras), University of Toronto; Lunenfeld-Tanenbaum Research Institute at Mount Sinai Hospital (Abe, Colwill, Gingras), Sinai Health System; Sunnybrook Research Institute (Jiang), Temerty Faculty of Medicine, University of Toronto; Public Health Ontario (Bolotin); Dalla Lana School of Public
27. Earle KA, Ambrosino DM, Fiore-Gartland A, et al. Evidence for antibody as a protective correlate for COVID-19 vaccines. Vaccine 2021;39:4423-8.

28. Abbasi J. The flawed science of antibody testing for SARS-CoV-2 immunity. JAMA 2021;326:1781-2.

29. Krammer F. A correlate of protection for SARS-CoV-2 vaccines is urgently needed. Nat Med 2021;27:1147-8.

Health (Bolotin, Tran), University of Toronto; Department of Laboratory Medicine and Pathobiology (Tran), University of Toronto; Ontario Renal Network (Oliver, Blake, Hladunewich), Ontario Health; Li Ka Shing Knowledge Institute (Perl), Unity Health Toronto; Toronto, Ont.; BC Renal Agency (Atiquzzaman, Levin), Vancouver, BC; Division of Nephrology (Blake), Schulich School of Medicine \& Dentistry, Western University, London, Ont.

Contributors: Christopher Chan, Anne-Claude Gingras and Michelle Hladunewich share senior supervising authorship. Kevin Yau and Kento Abe contributed equally as co-first authors. Kevin Yau, Christopher Chan, Jeffrey Perl, Shelly Bolotin, Vanessa Tran, Anne-Claude Gingras and Michelle Hladunewich contributed to the conception and design of the work. All of the authors contributed to data acquisition, analysis and interpretation of data for the work. Kevin Yau, Christopher Chan, Kento Abe, Anne-Claude Gingras and Michelle Hladunewich drafted the manuscript. All of the authors revised it critically for important intellectual content, gave final approval of the version to be published and agreed to be accountable for all aspects of the work.

Content licence: This is an Open Access article distributed in accordance with the terms of the Creative Commons Attribution (CC BY-NC-ND 4.0) licence, which permits use, distribution and reproduction in any medium, provided that the original publication is properly cited, the use is noncommercial (i.e., research or educational use), and no modifications or adaptations are made. See: https://creativecommons.org/licenses/by-nc-nd/4.0/

Funding: This study was supported by the COVID-19 Immunity Task Force (grant No. 2122-HQ-000071), which is funded by the Government of Canada and the Oreopoulos/Baxter Home Dialysis Grant from the Division of Nephrology at the University of Toronto. The equipment used is housed in the Network Biology Collaborative Centre at the Lunenfeld-Tanenbaum Research Institute, a facility supported by Canada Foundation for Innovation funding (Grant CFI 33474), the Ontario Government, Genome Canada and Ontario Genomics (OGI-139). Anne-Claude Gingras is supported by the Canadian Institutes of Health Research (CIHR; FDN 143301) and a Canada Research Chair (Tier 1) in Functional Proteomics. She has received funding from Providence Therapeutics, CIHR (for SARS-CoV-2 studies) and the Ontario Research Fund for SARS-CoV-2. Karen Colwill assisted with the agreement and managed funding from Medivolve for initial development of an enzyme-linked immunosorbent assay at Sinai Health System, outside the submitted work. Adeera Levin has received funding from AstraZeneca, Certa, Otsuka, Reata, Retrophin, The George Institute, Boeingher Ingelehem, CIHR, Janssen, Kidney Foundation of Canada, Merck, Oxford Clinical Trials, Merck, Amgen, Bayer, National Institutes of Health, National Institute of Diabetes and Digestive and Kidney Diseases, Chinook Therapeutics, and Johnson and Johnson.

Data sharing: All or portions of the de-identified data are available upon reasonable request to the corresponding author, Michelle Hladunewich (michelle.hladunewich@sunnybrook.ca).

Acknowledgements: The antibody measurements and initial data analysis were performed by Bhavisha Rathod, Mahya Fazel-Zarandi, Jenny Wang and Adrian Pasculescu. We obtained convalescent serum data from the Toronto Invasive Bacterial Diseases Network (REB studies No. 20-044, Unity Health; No. 02-0118-U/05-0016-C, Mount Sinai Hospital).

Accepted: Jan. 10, 2022

Correspondence to: Michelle Hladunewich, michelle.hladunewich@sunnybrook.ca 